UCRL-JC-121437

PREPRINT

CONF-951155-92

\title{
Electronic Structure Calculations of Calcium Silicate Hydrates
}

\author{
P.A. Sterne \\ A. Meike
}

November 1995 Boston, MA

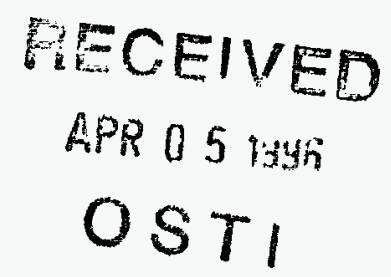

This paper was prepared for submittal to the Materials Research Society 1995 Fall Meeting

November 27-December 1, 1995

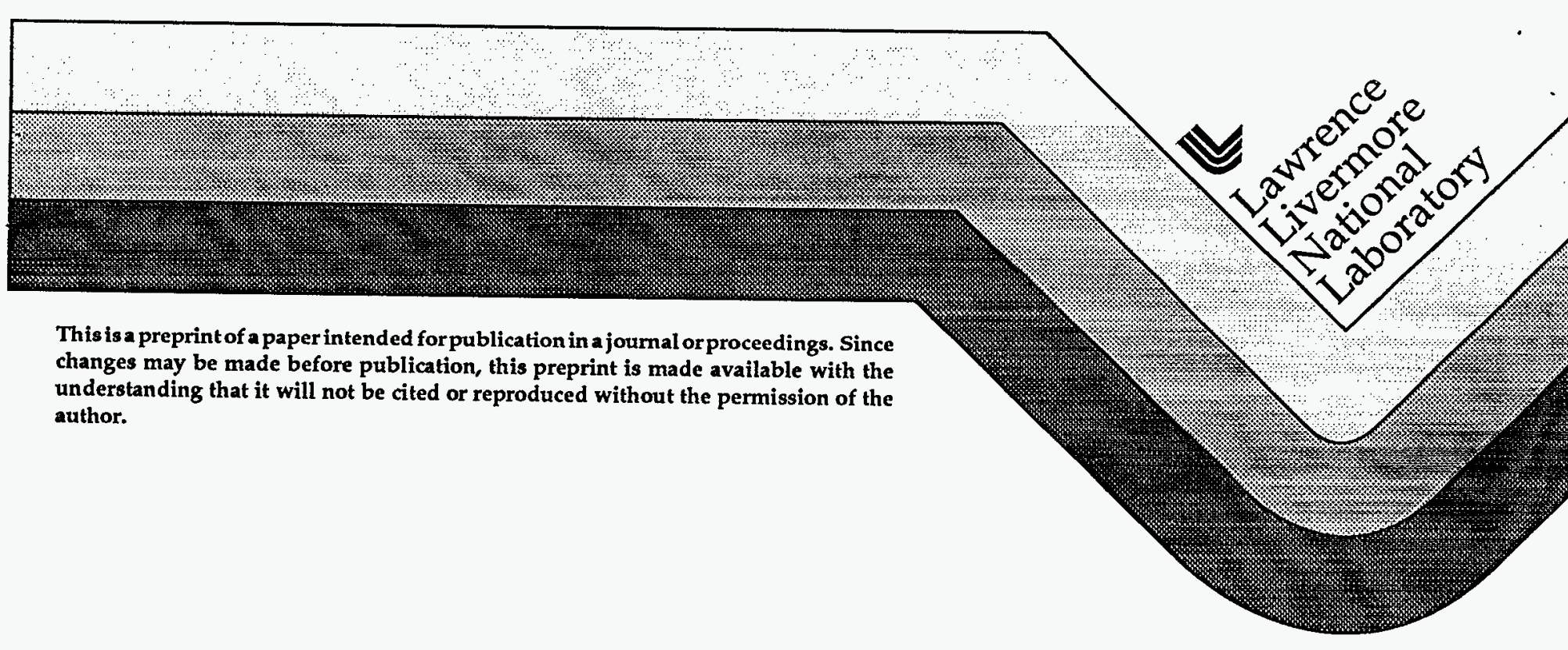




\section{DISCLAIMER}

This document was prepared as an account of work sponsored by an agency of the United States Government. Neither the United States Government nor the University of California nor any of their employees, makes any warranty, express or implied, or assumes any legal liability or responsibility for the accuracy, completeness, or usefulness of any information, apparatus, product, or process disclosed, or represents that its use wouldnot infringeprivately owned rights. Reference herein to any specific commercial products, process, or service by trade name, trademark, manufacturer, or otherwise, does not necessarily constitute or imply its endorsement, recommendation, or favoring by the United States Government or the University of California. The views and opinions of authors expressed herein do not necessarily state or reflect those of the United States Government or the University of California, and shall not be used for advertising or product endorsement purposes. 


\section{DISCLAMMER}

Portions of this document may be illegible in electronic image products. Images are produced from the best available original document. 


\title{
Electronic Structure Calculations of Calcium Silicate Hydrates
}

\author{
P.A. STERNE ${ }^{\dagger *}$, A. MEIKE* \\ † Department of Physics, University of California, Davis, CA 95616 \\ ${ }^{*}$ Lawrence Livermore National Laboratory, Livermore, CA 94551
}

\begin{abstract}
Many phases in the calcium-silicate-hydrate system can develop in cement exposed over long periods of time to temperatures above $25^{\circ} \mathrm{C}$. As a consequence, chemical reactions involving these phases can affect the relative humidity and water chemistry of a radioactive waste repository that contains significant amounts of cement. In order to predict and simulate these chemical reactions, we are developing an internally consistent database of crystalline $\mathrm{Ca}-\mathrm{Si}$-hydrate structures. The results of first principles electronic structure calculations on two such phases, wollastonite $\left(\mathrm{CaSiO}_{3}\right)$ and xonotlite $\left(\mathrm{Ca}_{6} \mathrm{Si}_{6} \mathrm{O}_{17}(\mathrm{OH})_{2}\right)$, are reported here. The calculated ground state properties are in very good agreement with experiment, providing equilibrium lattice parameters within about $1-1.4 \%$ of the experimentally reported values. The roles of the different types of oxygen atoms, which are fundamental to understanding the energetics of crystalline $\mathrm{Ca}-\mathrm{Si}$-hydrates are briefly discussed in terms of their electronic state densities. The good agreement with expuriment for the lattice parameters and the consistency of the electronic density of states features for the two structures demonstrate the applicability of these electronic structure methods in calculating the fundamental properties of these phases.
\end{abstract}

\section{INTRODUCTION}

Accurate predictions for the long term behavior of cement require the determination of kinetic and thermodynamic properties for a large range of possible hydrated mineral phases, i.e. mineral phases with varying amounts of structural $\mathrm{OH}$ and/or $\mathrm{H}_{2} \mathrm{O}$. A wide variety of phases can form in cement as it ages [1]. Although thermodynamic data are available for some hydrate phases, those for the calcium-silicate-hydrate family of phases are sparse [2]. These data are required as input for geochemical models, such as EQ3/6 [3]. Traditionally, geochemical models have been based on empirical parameters obtained experimentally from relatively large amounts $(10-20 \mathrm{~g})$ of pure phases [4]. Experimental values for the $\mathrm{Ca}$-Si-hydrate phases have been difficult to obtain, since they occur naturally only in small amounts and usually in mixed phases. Synthesis, characterization, and measurement of pure phases are difficult due to the ease with which structural water is gained and lost. Consequently, theory has a potentially important role to play, both in determining parameters that may be difficult to obtain from experiment, and in identifying the processes and phases that may need to be considered in the model. Consistency between theory and experiment also enhances our understanding of the physical and chemical processes involved in the aging of cements, and increases our confidence in the model's description of this aging process. Electronic structure calculation methods offer a means of obtaining important parameters such as the heats of formation from first principles, quantum-mechanical based calculations, and provide a valuable theoretical approach for 
complementing and cross-validating the often contradictory thermodynamic and crystal structural experimental data.

Electronic structure calculations based on density functional theory have been remarkably successful in predicting and describing the properties of metals, semiconductors, and simple insulators [5]. These calculations have been used to calculate explicitly the lowest energy phase from a set of candidate structures [6], but they can also be used to investigate and identify the reasons for the stability of a given phase [7]. This is particularly important in applications where some tailoring of materials properties is desired, such as in the immobilization of nuclear waste. Although their use has been confined to small systems with typically less than 20 atoms in the unit cell, recent developments in algorithms and computer power have brought larger systems within the range of these first principles methods. In addition, our studies of the crystalline $\mathrm{Ca}-\mathrm{Si}$-hydrate phases have driven us to push and expand the limits of these calculational methods by developing approaches and algorithms to deal with characteristics of these materials, such as low crystalline symmetries, open structures, and uncertainties in hydrogen positions.

In this paper, we present the first part of an investigation into the properties of crystalline calcium-silicate-hydrates using electronic structure calculations. The large unit cells in these systems make them challenging calculations for first principles methods. We have chosen to initiate the work using the hydrate poor end-members wollastonite and xonotlite, since they are some of the smallest and best documented structures in the system, and thus promise relative ease in the complex and computationally demanding calculations. We focus here on the equilibrium properties of each phase, and the similarities and differences in their electronic structure. Future work will address the more difficult task of extracting reliable ground-state energy differences and heats of formation from these calculations, and incorporating these quantities into existing geochemical models, as well as addressing other crystalline phases to examine the effect of increasing $\mathrm{H}_{2} \mathrm{O}$ content. Our ultimate goal is to produce a set of internally consistent energy differences for a range of $\mathrm{Ca}-\mathrm{Si}$-hydrate phases on the basis of first principles calculations.

\section{METHOD}

The Linear Muffin Tin Orbital (LMTO) method used in these calculations is a relatively fast and accurate self-consistent field variational basis set method for calculating the electronic structure of a perfect crystal $[8,9]$. The method uses a numerical radial wavefunction together with an angular-momentum-based expansion about each atom to provide the best possible minimal set of basis functions. The angular-momentum representation is very convenient for interpreting the resulting electronic structure. The numerical radial function enables the muffin tin orbital to adapt to the atomic environment and orthogonalize to the core states, thereby reducing the Hamiltonian matrix to include the valence states alone. Changes in core states are included self-consistently using a Dirac equation bound state solver.

The LMTO method simplifies the calculation by approximating the unit cell volume with a set of spheres, each centered on an atom site. This atomic sphere approximation (ASA) [8] is convenient and highly accurate in the case of a close-packed materials (e.g. fcc metals), but is a poor approximation in the case of the very open chain silicate structures. To improve this, we add "empty" spheres at open sites far from the real atomic positions. 
We increase the variational freedom in the calculation by including orbitals on these sites, and significantly improve the packing fraction, and hence the representation of the unit cell volume, by introducing these empty spheres [10]. We have recently developed an automated procedure for determining the optimum empty sphere positions for any given crystal structure to deal with the low symmetry structures that are of interest here [11].

The electron-electron interaction is treated using the local density approximation (LDA) [12] to density functional theory. This approximation is used almost universally in electronic structure calculations on periodic systems. Equilibrium lattice parameters are generally calculated to be within about $2-3 \%$ of experiment (6-9\% for the equilibrium volume), and elastic parameters like bulk moduli are generally within about $10 \%$ of experiment [5], both of which are remarkably accurate considering that no experimental input other than the crystal structure and the atomic numbers of the atoms are used. Further refinements of the calculations can be made both by relaxing atomic positions based on calculated forces to achieve the lowest possible energy state, and by verifying predicted physical properties against independent experimental measurements (e.g. NMR data, bulk modulus). It should be noted that the one-electron energy gap in LDA calculations generally underestimates the true experimental energy gap, and the theory makes no claims for the accuracy of the one-electron spectrum [13]. Nevertheless, the one-electron spectrum provides valuable insight into the nature of bonding and the origin of structural stability, and also provides a useful guide for interpreting experimental spectra that probe the electronic density of states, such as photoemission [14] and X-ray absorption [15].

The calculations have been performed using the crystal structures determined by Mamedov and Belov. Wollastonite $\left(\mathrm{CaSiO}_{3}\right)$ has a triclinic crystal structure with a 30 atom unit cell [16]. Seventeen empty spheres were added to fill space more efficiently. Xonotlite $\left(\mathrm{Ca}_{6} \mathrm{Si}_{6} \mathrm{O}_{17}(\mathrm{OH})_{2}\right)$ has a monoclinic structure with a 62 atom unit cell, excluding $\mathrm{H}$ [17]. Sixteen empty spheres were added to fill space. Since the exact positions of the H atoms are undetermined, they were placed in the empty sphere sites closest to the identified position of the $(\mathrm{OH})$ group in the xonotlite structure. The basis set for variational calculations includes s- and p- valence states on all sites containing real atoms (including $\mathrm{H}$ ), and sstates alone on the empty spheres. Brillouin zone integrations were performed on a grid of $64 \mathrm{k}$-points in the irreducible wedge for wollastonite, and $80 \mathrm{k}$-points for xonotlite.

\section{RESULTS AND DISCUSSION}

Total energies for wollastonite and xonotlite have been calculated at a range of volumes around the experimental unit cell volume. The volume changes were applied by a rigid scaling of the lattice parameters; no relaxations due to changes in lattice parameters or atomic positions were considered. The resulting energy-volume values were fitted to a Murnaghan equation of state. For wollastonite, the total energy minimum occurred at a volume of 2563.6 a.u., about 3.2\% smaller than the experimental value of 2647.3 a.u., and the bulk modulus was 1.99 Mbar. Similarly good agreement was found for xonotlite, with the total energy minimum at 5490.7 a.u., about $4.2 \%$ below the experimental value of 5729.8 a.u., and a bulk modulus of $1.9 \mathrm{Mbar}$. These correspond to agreement in the 1-1.4\% range for the lattice parameters. Since the calculations correspond to equilibrium structures at zero temperature, this good agreement with experiment can be further improved by accounting for thermal lattice expansion. These encouraging results confirm the applicability of the calculational method to these mineral phases. 


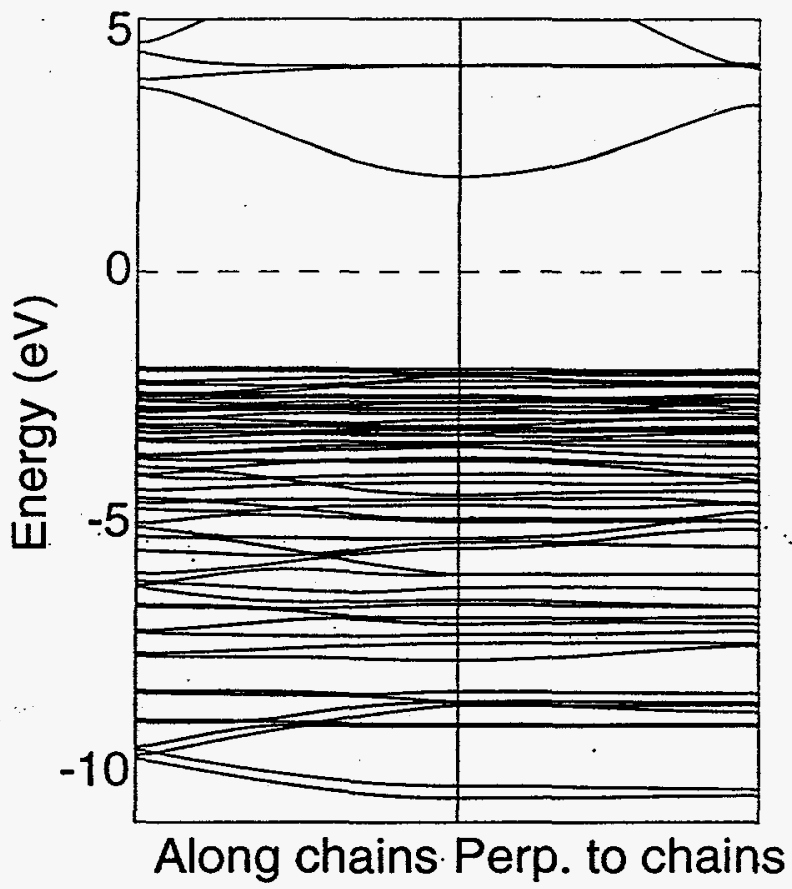

Figure 1: Electronic energy bands for wollastonite both along the silicate chains (left panel), and perpendicular to the chains (right panel). The $\Gamma$ point is at the center. The energy zero is chosen to be at the center of the energy gap.

Ideally we would like to be able to compare the total energies of the two different structures and deduce a heat of formation, which should require either full convergence in basis functions and $\mathrm{k}$-points in both calculations, or an equivalence between two calculations so that a cancellation of systematic errors can be performed. Unfortunately, the former option requires an extraordinary amount of computational time. The latter option requires sufficient differences in the details of the calculations that it is beyond the scope of this paper, but will be the focus of future work.

Even at this initial stage, the details of the electronic structure provide considerable insight into the nature of the bonding in the system. The energy bands in Figure 1 are plotted along two directions in the Brillouin zone, corresponding to states running parallel and perpendicular to the silicate chains in the crystal structure. The dispersion seen in the energy bands along the chains around $-10 \mathrm{eV}$ and $-6 \mathrm{eV}$ is indicative of $\mathrm{Si}-\mathrm{O}$ covalent bonding, while the much flatter bands seen through most of the valence band correspond to electrons that are localized in these directions, in this case mostly ionic-like oxygen states. .

Figure 2 shows the total density of electronic states for wollastonite, and its decomposition by atomic species obtained by projecting the charge density contribution for each electron state. The valence states are dominated by the contribution from the oxygen sites, with only small contributions coming from the $\mathrm{Ca}$ and Si sites. The Ca contribution is negligible throughout the valence band, in keeping with our expectation that the $\mathrm{Ca}$ ion is in a $2+$ state. The small contribution from $\mathrm{Si}$ is most prominent at lower energies, mainly in the regions where dispersive bands were observed in the band structure. This suggests that these states arise from $\mathrm{Si}-\mathrm{O}$ hybridization along the silicate chain. The large 


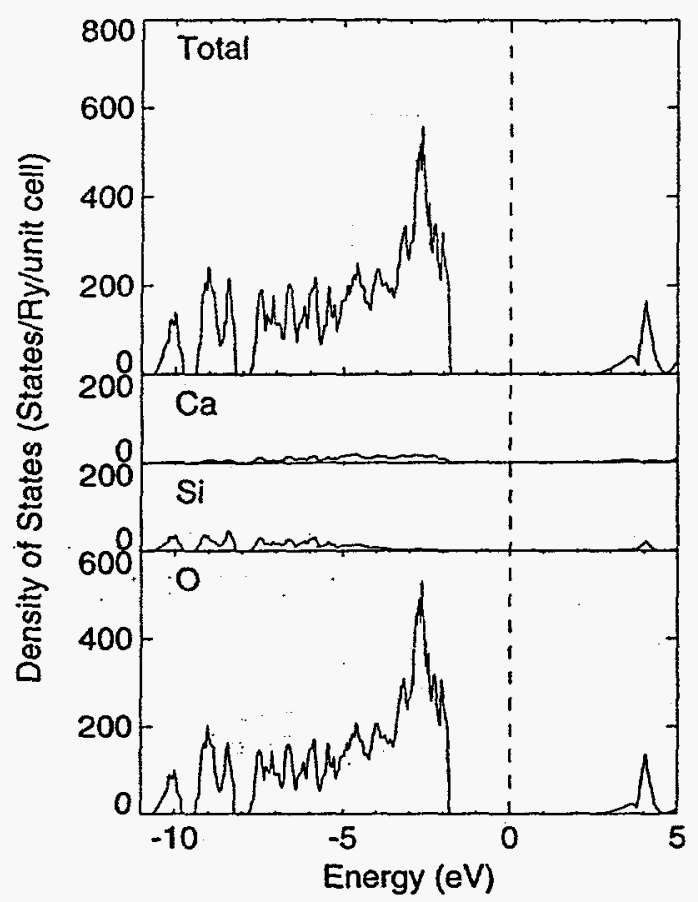

Figure 2: Electronic densities of states for wollastonite. Lower panels display decomposition by atomic species.

difference in the magnitude of the oxygen and silicon state densities in this energy region indicates that there is also a significant ionic character to these bonds.

There are nine inequivalent oxygen sites in the wollastonite crystal structure. For six of these sites, the oxygen atoms form bonding polyhedra around the $\mathrm{Ca}$ atoms, with each oxygen close to a single $\mathrm{Si}$ atom. The electronic density of states for these six sites are all very similar, with a large peak lying just below the energy gap and very little density at lower energies. This is typical of states that are primarily ionic in character. The electrons on the ionic oxygen sites have little or no overlap with electronic states on neighboring atoms, hence the narrow dispersionless bands resulting in a large peak in the electronic density of states (Figure 3). The three remaining inequivalent oxygen sites are in Si-O-Si bridging positions, with the oxygen atom bonded to two $\mathrm{Si}$ atoms. Two of these oxygen sites have similar Si-O bond lengths, and we consider these to be "typical" bridging sites. The third "atypical" bridging site has Si-O bond lengths about $10 \%$ shorter than the other two bridging sites.

Densities of states for all three types of oxygen sites are shown in Figure 3. Note that the bridging oxygen atoms have significant density at lower energies, in contrast with the more ionic non-bridging sites. While the density of states for the two "typical" bridging sites are very similar, the atypical bridging site looks very different. This indicates the sensitive relation between the calculated electronic structure and the atomic positions in the unit cell. In this case, a $10 \%$ change in bond lengths led to a profound difference in the density of states, which, in turn, has a significant effect on the calculated total energy, which depends primarily on the sum over occupied one-electron states. The sensitive dependence of the total energy on the atomic positions indicates that care must be taken in devising models based on the energies of simplified structural units, such as $\mathrm{Ca}-\mathrm{O}$ bonding polyhedra. The 


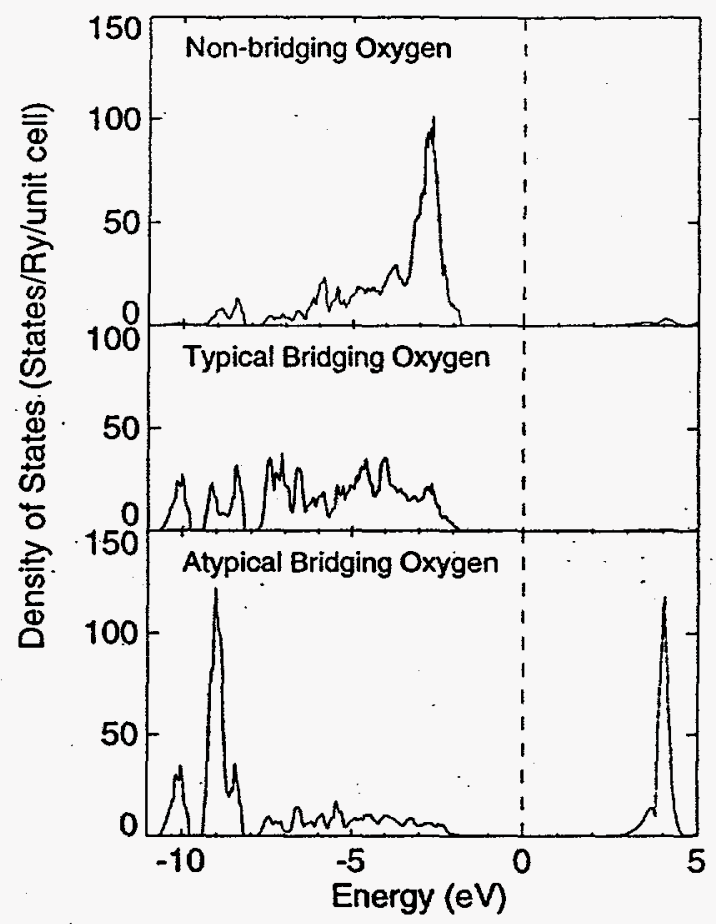

Figure 3: Partial electronic densities of states for three different oxygen sites in wollastonite. Top panel shows a typical non-bridging oxygen site, middle panel shows a typical bridging site, and the lowest panel shows an atypical bridging site.

energies associated with an individual structural unit, and the interaction energies between these structural units, may depend sensitively on the orientation, coordination, and strain of these units. A more comprehensive set of first principles calculations can quantify these effects, and place such simplified models on a more secure theoretical foundation.

Xonotlite shares many structural features with wollastonite, and this leads to strong similarities in the atom-decomposed density of states for many of the sites. A comparison of figure 3 and the left panel of figure 4 shows that the non-bridging xonotlite oxygens all have an ionic character similar to that seen in wollastonite, and the bridging atoms show the same sort of bonding features at lower energies, reflecting the similarities in the silicate chain in the two structures. The primary difference in the xonotlite structure is associated with the $\mathrm{OH}$ group, which is not present in wollastonite. The right panel in figure 4 shows the density of states for the oxygen and hydrogen atoms in this xonotlite $\mathrm{OH}$ group. Note that the oxygen has a strongly ionic character, similar to the non-bridging oxygen atoms. The small hydrogen contribution throughout the valence bands probably reflects the overlap of orbitals from neighboring sites into the relatively large volume of the empty site occupied by the $\mathrm{H}$ atom, and does not represent states derived primarily from hydrogen-related orbitals. The peak in the unoccupied states just above the valence band maximum is of hydrogen character, however, and represents an electronic state induced by hydrogen in the energy gap of the chain silicate structure. This energy level is likely to depend strongly on the position of the $\mathrm{H}$ atom, and so may serve as a means of probing the location of the $\mathrm{H}$ atom in the unit cell. This direction will be explored in more depth as we calculate the electron density of states of the more hydrated members of 

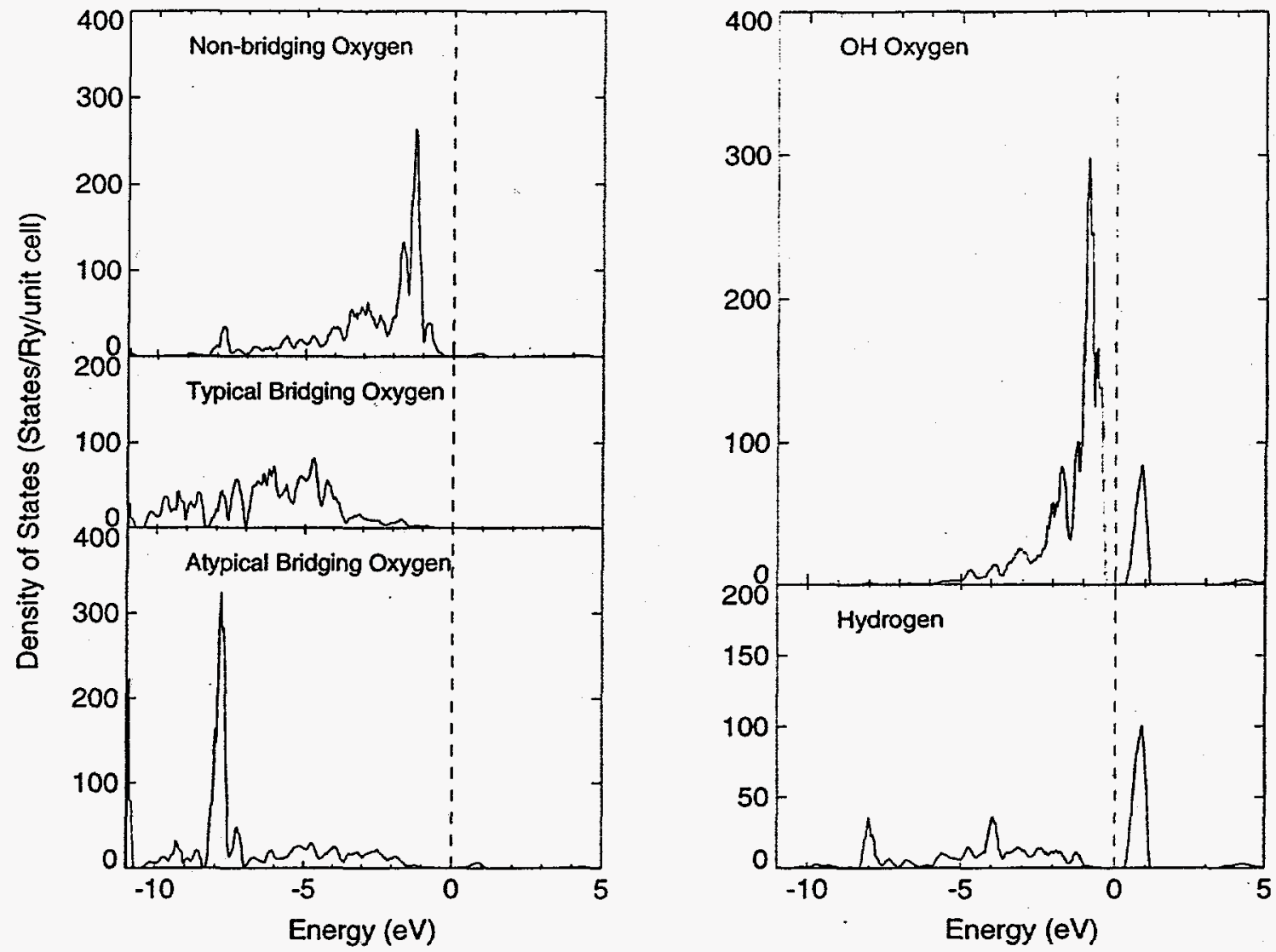

Figure 4: Electronic densities of states for oxygen and hydrogen sites in xonotlite. The left panel shows the state densities for the non-bridging, typical bridging: and atypical bridging oxygen sites. These resemble the state densities for the similarly located atoms in the wollastonite structure (Fig. 3). The right panel shows the density of states for the $\mathrm{OH}$ group in xonotlite, with the oxygen state density in the upper right panel and the hydrogen density of states in the lower right panel.

the $\mathrm{Ca}-\mathrm{Si}$-hydrate system, especially those that contain structural $\mathrm{H}_{2} \mathrm{O}$. Ultimately, the theoretical exploration of electron density of states of oxygen, hydroxyl and $\mathrm{H}_{2} \mathrm{O}$ groups, combined with experimental studies, will help us to understand and predict hydration and dehydration phenomena in these phases.

\section{CONCLUSIONS}

The ultimate goal of this research is to determine the energetics of hydration for crystalline calcium-silicate-hydrate phases which are found both naturally and in synthetic systems, including cementitious materials exposed to elevated temperature. The work presented here represents an initial step in this direction. We have demonstrated that these electronic structure calculations give reliable results, even for the relatively large wollastonite and xonotlite unit cells considered here. Lattice parameters are in good agreement with experiment, and the general features of the electronic structure can be readily understood in terms of the underlying atomic positions. We have demonstrated, through a 
detailed discussion of some aspects of the electronic structure of these phases, that electronic structure calculations offer a new and potentially fruitful approach to their study. This is true not only in terms of their ability to calculate important parameters such as heats of formation, which can ultimately be used to predict chemical reactions over long time periods, but also by virtue of the insight that they can provide into the nature of hydration and dehydration in minerals.

\section{ACKNOWLEDGMENTS}

This work was performed under the auspices of the U.S. Department of Energy by Lawrence Livermore National Laboratory under contract number W-7405-ENG-48. Financial support for this project was obtained from the Fundamental Materials Investigations activity of Subsidiary Agreement No. 2 between Atomic Energy of Canada Ltd. and the U.S. Department of Energy as part of the Civilian Radioactive Waste Management Program.

\section{REFERENCES}

[1] H.F.W. Taylor, The Chemistry of Cements (Academic Press, New York, 1964).

[2] C.J. Bruton, B.L. Phillips, A. Meike, S. Martin, and B.E. Viani, Mat. Res. Soc. Symp. Proc. Vol. 333, 327 (1994).

[3] T.J. Wolery, Lawrence Livermore National Laboratory UCRL-MA-110662, Pt. 1 (1992).

[4] J.W. Johnson, E.H. Oelkers, and H.C. Helgeson, Computers and Geosciences 18, 899 (1992).

[5] U. von Barth and A.R. Williams, in Theory of the Inhomogeneous Electron Gas, edited by S. Lundqvist and N.H. March (Plenum, New York, 1983).

[6] M.T. Yin and M.H. Cohen, Phys. Rev. B 26, 5668 (1982).

[7] See, e.g. J.F. Clark, F.J. Pinski, D.D. Johnson P.A. Sterne, J.B. Staunton, and B. Ginatempo, Phys. Rev. Lett. 74, 3225 (1995).

[8] O.K. Anderson, Phys. Rev. B 12, 3060 (1975).

[9] H.L. Skriver, The LMTO Method, Vol. 41 of Springer Series in Solid State Sciences (Springer, New York, 1984).

[10] D. Glötzel, B. Segall, and O.K. Anderson, Solid State Commum. 36, 403 (1980).

[11] P.A. Sterne and J. van Ek (unpublished).

[12] W. Kohn and P. Vashista, in Theory of the Inhomogeneous Electron Gas, edited by S. Lundqvist and N.H. March (Plenum, New York, 1983).

[13] R.O. Jones and O. Gunnarsson, Rev. Mod. Phys. 61, 689 (1989).

[14] E. Tamura, G.D. Waddill, J.G. Tobin, and P.A. Sterne, Phys. Rev. Lett. 73, 1533 (1994).

[15] J. van Ek, E. Tamura, M. Froba, and J. Wong, Phys. Rev. Lett. 74, 4899 (1995).

[16] K.S. Mamedov and N.V. Belov, Dokl. Acad. Nauk. SSSR, 107, 463 (1956).

[17] K.S. Mamedov and N.V. Belov, Dokl. Acad. Nauk. SSSR, 104, 615 (1955). 